\title{
Estabilidad de espumas formuladas con proteínas de soja tratadas a pH ácido
}

\author{
Abirached, C. ${ }^{(1)}$, Medrano, C. A. ${ }^{(1)}$, Panizzolo, L. A. ${ }^{(1)}$, Moyna, P. ${ }^{(1)}$, Añón, M. C. ${ }^{(2)}$ \\ (1)Departamento de Ciencia y Tecnología de los Alimentos, Facultad de Química, Universidad de la República, Uruguay \\ ${ }^{(2)}$ Centro de Investigación y Desarrollo en Criotecnología de Alimentos, Universidad Nacional de La Plata, Argentina. \\ Contacto: abirached@fq.edu.uy
}

Recibido: 07/04/2010 - Aprobado: 04/11/2010

\begin{abstract}
$\underline{\text { Resumen }}$
Se estudió la influencia del tratamiento a $\mathrm{pH}$ ácido de aislados proteicos de soja sobre las propiedades espumantes mediante el análisis de las constantes cinéticas de desproporción y drenado y el relacionamiento de éstas con los parámetros de reología interfacial. Los aislados proteicos de soja fueron obtenidos a partir de harina desgrasada. Una porción se llevó a pH 8,0 y otra porción se trató a pH 2,0. De la porción a pH 2,0 una parte se dejó a este pH y otra parte se neutralizó a pH 8,0. Todas las porciones se liofilizaron. Para la identificación y caracterización estructural de las muestras se realizaron análisis de electroforesis desnaturalizante, calorimetría diferencial de barrido, solubilidad e hidrofobicidad superficial. Se obtuvieron espumas por el método de burbujeo de gas. Se determinó la velocidad inicial de pasaje de líquido a la espuma y el volumen máximo de líquido incorporado a la espuma. El proceso de desestabilización de las espumas formadas se analizó ajustando los datos obtenidos a una cinética de segundo orden bifásica. Se realizaron estudios de reología interfacial con un tensiómetro de gota. El tratamiento a pH ácido mejoró tanto la espumabilidad como la estabilidad de las espumas. La proporción de líquido drenado por escurrido gravitatorio fue significativamente superior al volumen drenado debido a la desproporción. El tratamiento a pH 2,0 redujo la desestabilización por desproporción de Ostwald, lo que sugiere la formación en la interfase de una película más cohesiva, que se confirma con los parámetros de reología interfacial.
\end{abstract}

Palabras clave: Drenado, desproporción de Ostwald.

\begin{abstract}
$\underline{\text { Abstract }}$
In this work the influence of the acid pH treatment of soy protein isolate on the foaming properties by analysis of the kinetic constants of disproportion and drainage and their relation with the interfacial rheology parameters was studied. Soy protein isolates were obtained from defatted flour. A portion was brought to $\mathrm{pH} \mathrm{8.0,} \mathrm{another} \mathrm{portion} \mathrm{was} \mathrm{treated} \mathrm{at} \mathrm{pH} 2.0$. From the portion treated at $\mathrm{pH} 2.0$ a portion was left at this $\mathrm{pH}$ and another was neutralized to $\mathrm{pH} 8.0$. All portions were lyophilized. For the identification and structural characterization of the samples were performed denaturing electrophoresis analysis, differential scanning calorimetry, solubility and surface hydrophobicity. Foams were obtained by the method of gas bubbling. The initial rate of passage of liquid to foam and the maximum volume of fluid incorporated to the foam were determined. The process of destabilization of foams formed was analyzed by fitting the data to a biphasic second-order kinetics. Studies were performed with an interfacial rheology drop tensiometer. Acid pH treatment improved both the foam and foam stability. The proportion of liquid drained by gravity was significantly higher than the drained volume because of the disproportion. Treatment at pH 2.0 reduced the Ostwald disproportion destabilization, suggesting the formation at the interface of a more cohesive film, which is confirmed by the interfacial rheology parameters.

Keywords: Drainage, Ostwald ripening.
\end{abstract}

\section{Introducción}

En el presente, las proteínas de soja se usan cada vez más en la elaboración de productos alimenticios fabricados como emulsificantes, agentes espumantes, espesantes y agentes de absorción y retención de agua. Sin embargo, el óptimo uso de la proteína de soja en, por ejemplo, emulsiones y productos alimenticios tipo espuma requiere un conocimiento de sus propiedades de superficie bajo varias condiciones mucho mayor del que está actualmente disponible (Utsumi et al., 1997; Martin et al., 2002). Para estudiar las propiedades de superficie se analizan los parámetros viscoelásticos, módulo dilatacional superficial, E, y sus componentes elástica, Ed, y viscosa, Ev.

Las espumas son dispersiones de burbujas de aire en un medio líquido que contiene un agente activo de superficie, también llamado agente espumante. Este agente activo de superficie tiende a situarse en la superficie, protegiendo del colapso de las burbujas. La composición y las propiedades de la capa adsorbida determinan la estabilidad y propiedades físicas resultantes de la espuma (Maldonado-Valderrama et al., 2007). El proceso de desestabilización de una espuma consiste en la tendencia de la fase gaseosa discontinua a formar una fase continua por aproximación y fusión de las burbujas, a fin de alcanzar un área superficial mínima (mínima energía libre). A este proceso se opone la película proteica superficial, que como barrera mecánica es más efectiva cuanto mayor son su viscoelasticidad y su rigidez. Los mecanismos de desestabilización de una espuma son: drenaje de líquido por efecto de la gravedad, desproporción o maduración 
de Ostwald, por el que las burbujas grandes crecen a expensas de las pequeñas por difusión de gas a través de la lamela, y colapso de la espuma por ruptura de las lamelas. Todos estos mecanismos ocurren simultánea y sinérgicamente (Wagner, 2000). Se han realizado varios estudios de la cinética de desestabilización de espumas. Panizzolo (2005) plantea que existen dos procesos diferenciados de drenado de líquido de la espuma, uno debido al drenado de líquido propiamente dicho y otro a la desproporción de Ostwald. En base a ello planteó un modelo de segundo orden bifásico donde se determinan las constantes de velocidad y los volúmenes máximos drenados debido al drenado gravitacional y a la desproporción.

La viscosidad dilatacional de superficie es crucial para la capacidad de un sistema tensioactivo para formar una espuma estable. La viscosidad superficial disminuye las distorsiones mecánicas que de otro modo podrían causar una ruptura de las lamelas de las espumas (Koelsch y Motschmann, 2005). Por su parte, Georgieva et al. (2009) plantearon que la ruptura de la película es controlada por elasticidad dilatacional superficial y que la maduración de Ostwald es principalmente afectada por el espesor de la película entre las burbujas y la solubilidad del gas en el agua.

Después de la disolución en agua o a un pH ligeramente alcalino, las proteínas de soja pueden separarse en varias fracciones por cromatografía permeable de gel, electroforesis, ultracentrífuga, etcétera. Cuando se utiliza esta última técnica se separan cuatro fracciones cuyos coeficientes de sedimentación S20,w (unidades Svedberg, a $20^{\circ} \mathrm{C}$, en el agua) son respectivamente iguales a 2, 7, 11 y 15. Las globulinas glicinina (11S) y $\beta$-conglicinina (7S) representan por sí solas más del $70 \%$ de las proteínas del grano de soja. La $\beta$-conglicinina es una glicoproteína que contiene en torno de $5 \%$ de glúcidos (mannosa y $\mathrm{N}$-acetil-glucosamina). Está constituída por tres subunidades $\alpha$, $\alpha^{\prime}$ y $\beta$, de carácter ácido (Cheftel, 1989). La glicinina es una proteína globular y se presenta como un hexámero. Las seis subunidades monoméricas tienen la estructura general $\mathrm{AB}$, donde A representa un polipéptido ácido y $\mathrm{B}$ un polipéptido básico. Las cadenas A y B están unidas por un puente disulfuro. Dependiendo de la solubilidad, $\mathrm{pH}$ y fuerza iónica la glicinina (11S) se disocia en la forma $7 \mathrm{~S}\left((\mathrm{AB})_{3}\right)$ y/o en la forma $3 \mathrm{~S}(\mathrm{AB})$. A pH 6,7 la glicinina está presente en las formas $3 \mathrm{~S}, 7 \mathrm{~S}$ y $11 \mathrm{~S}$ en 0,3 y $57 \%$, respectivamente y a $\mathrm{pH}$ 3,0 en 70,27 y $0 \%$, respectivamente. Esto significa que a $\mathrm{pH} 3,0$ una mezcla de las formas $3 \mathrm{~S}$ y $7 \mathrm{~S}$ está presente y a $\mathrm{pH} 6,7$ está presente principalmente la forma $11 \mathrm{~S}$. Aunque tanto las formas $3 \mathrm{~S}$ como $11 \mathrm{~S}$ son capaces de formar una red en la interfase aire-agua, la red de $11 \mathrm{~S}$ es menos rígida (fuerte). La diferencia en la rigidez reside en la estructura más compacta de la forma 11S y la ocurrencia de otros tipos de interacciones dentro de las moléculas. La forma $3 \mathrm{~S}$ es más flexible debido a la alta repulsión electrostática dentro de la molécula. Por lo tanto, será más fácil de desplegar durante la adsorción en la interfase y tendrá más posibilidades de formar enlaces físicos y covalentes intermoleculares (Martin et al., 2002).

Cualquier cambio de $\mathrm{pH}$, fuerza iónica, temperatura, composición del solvente, etcétera, en el entorno de una proteína nativa, obligará a la molécula a asumir una nueva estructura de equilibrio. Los cambios significativos en las estructuras secundaria, terciaria y cuaternaria sin ruptura de los enlaces peptídicos de la cadena son considerados como "desnaturalización". En algunos casos la desnaturalización de las proteínas es deseable. Las proteínas parcialmente desnaturalizadas son más digestibles y tienen mejores propiedades de formación de espuma y emulsión que las proteínas nativas (Damoradan, 2008).

Por lo tanto, el objetivo de este trabajo fue estudiar la influencia del tratamiento a $\mathrm{pH}$ ácido de aislados proteicos de soja sobre las propiedades espumantes, mediante el estudio de las constantes cinéticas de desproporción y drenado y el relacionamiento de éstas con estudios de reología interfacial.

\section{Materiales y Métodos}

\section{Obtención y tratamiento con pH de las proteínas de soja}

El aislado proteico de soja en estado nativo (APSn) se obtuvo por solubilización acuosa de harina de soja desengrasada en medio alcalino $(\mathrm{pH} 8,0)$ y posterior precipitación a $\mathrm{pH} 4,5$, dispersión del precipitado en medio alcalino ( $\mathrm{pH} \mathrm{8,0)} \mathrm{y} \mathrm{secado} \mathrm{por} \mathrm{liofilización} \mathrm{(Petruccelli} \mathrm{y}$ Añón, 1994). Con las proteínas nativas se preparó una dispersión de 15 $\mathrm{mg} / \mathrm{ml}$, se la llevó a pH 2,0 con $\mathrm{HCl}$ 6,0 N durante 1 hora y se liofilizó (APSt2). Con estas proteínas tratadas con $\mathrm{pH}$ ácido, se preparó una dispersión de $15 \mathrm{mg} / \mathrm{ml}$, se la llevó a pH 8,0 con $\mathrm{NaOH} \mathrm{5,0} \mathrm{N} \mathrm{durante}$ una hora y se liofilizó (APSt2-8).

Se determinó contenido de proteína por método de Lowry (Lowry et al., 1951) en cada etapa.

\section{Identificación de las fracciones por electroforesis}

Las corridas electroforéticas se realizaron en un equipo BIORAD MINI PROTEIN II CELL. Se llevó a cabo una electroforesis desnaturalizante con SDS en gradiente $7-15 \%$ de arcrilamida, según lo descrito por Petruccelli y Añón (1994). La corrida fue hecha a un voltaje constante de $90 \mathrm{~V}$ para dos geles de $1,0 \mathrm{~mm}$ de espesor. Se utilizó una unidad de electroforesis SE 640 Hoefer Scientific instruments. El peso molecular de las proteínas fue estimado mediante un patrón de pesos moleculares LMW Pharmacia consistente en seis proteínas de peso molecular 14,4, 20,1, 30,0, 45,0, 66,0 y 97,0 kDa.

\section{Solubilidad}

Se determinó la solubilidad de las diferentes muestras dispersándolas en solución de fosfato de sodio $10 \mathrm{~mm}$ pH 8,0 al 0,1\% $\mathrm{p} / \mathrm{v}$ durante 60 minutos a temperatura ambiente con agitación constante. Posteriormente las muestras fueron centrifugadas a $10.000 \mathrm{xg}$ durante 10 minutos a $4{ }^{\circ} \mathrm{C}$. El contenido de proteína en el sobrenadante se determinó utilizando el método de Lowry (Lowry et al., 1951).

\section{Hidrofobicidad superficial}

Los valores de hidrofobicidad aromática superficial $\left(\mathrm{H}_{0}\right)$ de cada muestra fueron determinados por medio de la sonda fluorescente ANS (ácido 8-anilino 1 naftalen-sulfónico) de acuerdo al método de Hayakawa y Nakai (1985). La hidrofobicidad superficial se determinó como la pendiente inicial de la curva de intensidad de fluorescencia relativa porcentual versus la concentración de proteína según Kato y Nakai (1980).

\section{Estabilidad térmica y grado de conformación nativa mediante calorimetría diferencial de barrido (DSC)}

Se determinó la temperatura, entalpía de desnaturalización $(\Delta \mathrm{Hd})$, utilizando un Calorímetro de Barrido Diferencial - DSC Serie Q100 TA Instruments con software Universal Analysis 2000. Las muestras analizadas fueron dispersiones de APSn, APSt2, APSt2-8 previamente descriptos. Se utilizaron cápsulas de aluminio herméticas en las que se colocaron aproximadamente $20 \mathrm{mg}$ de muestra. Como referencia se 
utilizó una cápsula con muestra, previamente sometida al ensayo. Las condiciones del ensayo fueron un calentamiento a una velocidad de 10 ${ }^{\circ} \mathrm{C} / \mathrm{min}$ en un intervalo de temperatura entre 20 y $120^{\circ} \mathrm{C}$.

\section{Análisis de la capacidad para formar y estabilizar espumas}

El estudio de formación y estabilidad de espuma se realizó mediante el método conductimétrico desarrollado por Loisel et al (1993), con pequeñas modificaciones. La espuma fue generada por burbujeo de aire a través de una placa de vidrio sinterizado tipo G2. El ensayo se realizó con dispersiones de APSn y APSt2 en una concentración de proteína de $1 \mathrm{mg} / \mathrm{ml}$ en buffer fosfato de sodio 0,1 $\mathrm{M}, \mathrm{pH} 8,0$, fuerza iónica 0,28 y a pH 2,5 con fuerza iónica ajustada con $\mathrm{NaCl}$ a 0,28 . Cada medición se realizó al menos por triplicado.

Se determinó la velocidad inicial de pasaje de líquido a la espuma (Vo), mediante la pendiente inicial de la curva $\mathrm{V}_{\mathrm{LE}}$ vs $\mathrm{t}$ según Wagner et al. (1996) y el volumen máximo de líquido incorporado a la espuma $\left(\mathrm{V}_{\mathrm{LE}} \max \right)$.

La cinética de desestabilización de la espuma se estudia con la ecuación planteada por Panizzolo (2005):

$$
V(t)=\frac{V_{g}^{2} k_{g} t}{\left(V_{g} k_{g} t+1\right)}+\frac{V_{d}^{2} k_{d} t}{\left(V_{d} k_{d} t+1\right)}
$$

Donde: $\mathrm{V}(\mathrm{t})$ : volumen de líquido drenado a tiempo $\mathrm{t} ; \mathrm{Vg}$ : volumen máximo de líquido debido al proceso de drenado gravitacional Vd: volumen máximo de líquido drenado por la difusión de gas; $\mathrm{Kg}$ : constante de velocidad correspondiente al proceso de drenado gravitatorio; $\mathrm{Kd}$ : constante de velocidad correspondiente al proceso de difusión de gas o desproporción.

\section{Reología interfacial}

Las mediciones de las propiedades reológicas superficiales de las películas adsorbidas sobre la interfase (agua/aire) de todas las muestras se llevaron a cabo en un tensiómetro dinámico de gota (Tracker, IT-Concept, Saint-Clementtes Places, France).

Las propiedades dilatacionales de superficie, E (módulo dilatacional), Ed (elasticidad dilatacional) y Ev (elasticidad dilatacional de superficie), se midieron en función del tiempo. La amplitud y la frecuencia angular se mantuvieron constantes en $10 \%$ y $200 \mathrm{mHz}$, respectivamente. Los experimentos se realizaron a $20 \pm 1$ ${ }^{\circ} \mathrm{C}$. El pH y la fuerza iónica se mantuvieron constantes en 8,0 o 2,5 y 0,28 , respectivamente, utilizando un buffer fosfato de sodio $0,1 \mathrm{M}$. La solución de proteína de concentración $1 \mathrm{mg} / \mathrm{ml}$ se coloca en la cubeta A continuación, una gota de aire se agrega desde la jeringa y se deja reposar durante $120 \mathrm{~min}$ a $20^{\circ} \mathrm{C}$ para lograr la absorción de proteínas en la interfase aire-agua. Después de este tiempo se comenzó con la oscilación sinusoidal durante 60 minutos. Cada medición se hizo al menos por triplicado.

\section{Análisis estadístico}

El tratamiento estadístico de datos se realizó en todos los casos mediante análisis de la varianza (ANOVA) con $\alpha=0,05$ y la comparación de medias por la prueba de mínimas diferencias significativas (LSD) con $\alpha=0,05$, utilizando el programa Statgraphics plus 7.0.

\section{Resultados y Discusión}

La solubilidad disminuyó con el tratamiento ácido: 93 $\pm 8 \%$ para APSn y $90 \pm 5 \%$ para APSt 2 expresada como gramos de proteína soluble en $100 \mathrm{~g}$ de proteína; probablemente esto se deba a la formación de agregados insolubles luego del tratamiento ácido.

La hidrofobicidad superficial aumentó con el tratamiento a pH 2,0 del aislado proteico de soja, ya que al desnaturalizarse las proteínas exponen sus grupos hidrofóbicos: $27.4 \pm 0.3$ para APSn y $31.3 \pm 0.7$ para APSt2.

Se pudieron identificar por electroforesis las bandas de las proteínas $11 \mathrm{~S}$ (glicinina) y $7 \mathrm{~S}$ ( $\beta$-conglicinina) constituyentes del aislado proteico de soja.

En el análisis de DSC el APSn presentó dos picos endotérmicos, uno a $86 \pm 10{ }^{\circ} \mathrm{C}$ y otro a $105 \pm 2{ }^{\circ} \mathrm{C}$. El primero de ellos corresponde a la $\beta$-conglicinina y representa el $27 \pm 4 \%$ y el otro es la glicinina, que representa el $73 \pm 4 \%$ de aislado proteico de soja. En el termograma de APSt 2 no se encuentra el pico de la $11 \mathrm{~S}$ y el de la $7 \mathrm{~S}$ está a temperaturas más bajas y tiene una menor entalpía de desnaturalización $(\Delta \mathrm{Hd})$ en comparación con APSn. En el termograma de APSt2-8, sólo aparece el pico de la $7 \mathrm{~S}$ con un $\triangle \mathrm{Hd}$ mayor y a temperaturas más altas que para APSt2 (Gráfico 1 a y b). Estas diferencias podrían deberse a que se forma una estructura diferente a la nativa (evidenciado por diferencia en $\Delta \mathrm{H}$ ) o la recuperación de un porcentaje de la estructura nativa. Por lo tanto, la $\beta$-conglicinina es más resistente a la desnaturalización por $\mathrm{pH}$ y presenta recuperación de la conformación nativa (renaturalización) a diferencia de la glicinina (Puppo, 1997).

En general, se observó por los parámetros VLEmax y Vo que la espumabilidad del aislado mejoró con el tratamiento a pH 2,0 (Tabla 1). Al comparar VLEmax y Vo no se hallan diferencias significativas $(\alpha \leq 0,05)$ entre las espumas formadas con APSt2 dispersas en un medio a $\mathrm{pH} 2,5$ y a $\mathrm{pH} 8,0$. La mayor hidrofobicidad superficial de APSt2 contribuiría a la mejor espumabilidad.

Las espumas preparadas con APSn dispersa en solución acuosa a $\mathrm{pH}$ 8,0 presentaron mayores valores de las constantes de desestabilización kg y kd en comparación a las preparadas con APSt 2 tanto a pH 8,0 como a pH 2,5. Por lo tanto, el aislado proteico de soja mejora su estabilidad con el tratamiento ácido. Las espumas preparadas con APSt2 en medio a $\mathrm{pH} 2,5$ y $\mathrm{pH} 8,0$ no presentan diferencias significativas $(\alpha \leq 0,05)$ en los valores de $\mathrm{kg}$ y $\mathrm{kd}$, por lo que en el comportamiento del aislado no se representa el fenómeno de recuperación de parte de la estructura nativa de la $\beta$-conglicinina, probablemente por encontrarse ésta en una menor proporción (Tabla 1).

Los mecanismos de desestabilización también ocurren durante la formación de la espuma, por consiguiente, para que haya una buena formación de espuma, las proteínas deben ser capaces de contrarrestar estos mecanismos de desestabilización. Es lógico pensar que aquellas proteínas que proporcionen a las soluciones buenas características de espumabilidad tengan cualidades que les permitan contrarrestar los mecanismos de desestabilización. Esto concuerda con los resultados, ya que APSt 2 presenta mejor espumabilidad y estabilidad que APSn.

Con el fin de estudiar en qué medida contribuyen los mecanismos de drenado gravitacional y desproporción de Ostwald al total de volumen de líquido drenado, se determinaron las proporciones de volumen de líquido drenado mediante escurrido gravitatorio $(\mathrm{Vg})$ y de volumen drenado debido a la desproporción (Vd). En todos los casos ensayados la proporción de líquido drenado por escurrido gravitatorio fue significativamente superior (nunca menor de $80 \%$ ) al volumen drenado debido a la desproporción (Tabla 1). Para el cálculo de Vg y Vd se estableció la suposición de que todo el líquido drenó (tiempo infinito). Entonces, las espumas que incorporaron más líquido drenan 
más líquido, por lo que el $\mathrm{Vg}$ de las proteínas nativas tiene un valor menor que el de las tratadas con $\mathrm{pH}$ ácido. En cuanto a las proporciones de contribución de cada mecanismo, las espumas formadas con APSt2 tanto a pH 8,0 y pH 2,5 tienen una mayor proporción de drenado gravitacional y menor desproporción de Ostwald por tener tal vez una película más cohesiva. Esto se confirmará con los estudios de reología interfacial.

En cuanto al módulo dilatacional (E), no existen diferencias significativas entre APSn y APSt2 a pH 8,0: éstos son menores que para APSt2 a pH 2,5, lo que indicaría que en este caso se produce una película más resistente (Tabla 2). El componente dilatacional viscoso a $\mathrm{pH}$ 8,0 es mayor para APSt2 que para APSn, y esto podría explicar la mayor estabilidad de las espumas preparadas con APSt2 en relación a las preparadas con APSn a pH 8,0, ya que la viscosidad superficial disminuye las distorsiones mecánicas que pueden causar la ruptura de las lamelas de las espumas. Para APSt2 a pH 2,5 se observa un aumento de casi el doble del componente elástico con respecto a las correspondientes a APSn y APSt2 a pH 8,0, lo cual no se ve reflejado en $\mathrm{Kg}$ y $\mathrm{Kd}$, pero sí en los valores de Vg y Vd. APSt2 a pH 8,0 cuenta con un menor valor de Vd que APSt2 a pH 2,5 debido a que APSt2 a pH 8,0 presenta una mayor viscosidad dilatacional. La mayor viscosidad dilatacional evita la ruptura de la película y, por lo tanto, explica la menor proporción de drenado por desproporción de Ostwald (Vd) de las espumas formadas con APSt2 a pH 8,0. En cambio, para APSt2 a pH 2,5 la responsable de la estabilización de la película es la componente elástica. Una película estable requiere superficies elásticas para amortiguar las perturbaciones externas y, por ende, evitar la ruptura de la película (Stubenrauch y Miller, 2004). Esto puede deberse a que a $\mathrm{pH} 8,0$ la glicinina se encuentra como hexámero (11S) y a pH 2,5 como dímero (3S). La estructura más compacta de la forma $11 \mathrm{~S}$ y la ocurrencia de otros tipos de interacciones dentro de las moléculas hace que forme películas menos fuertes que la forma $3 \mathrm{~S}$, que es más flexible dada la alta repulsión electrostática dentro de la molécula. Al ser de mayor tamaño, la forma $11 \mathrm{~S}$ favorece la componente viscosa.

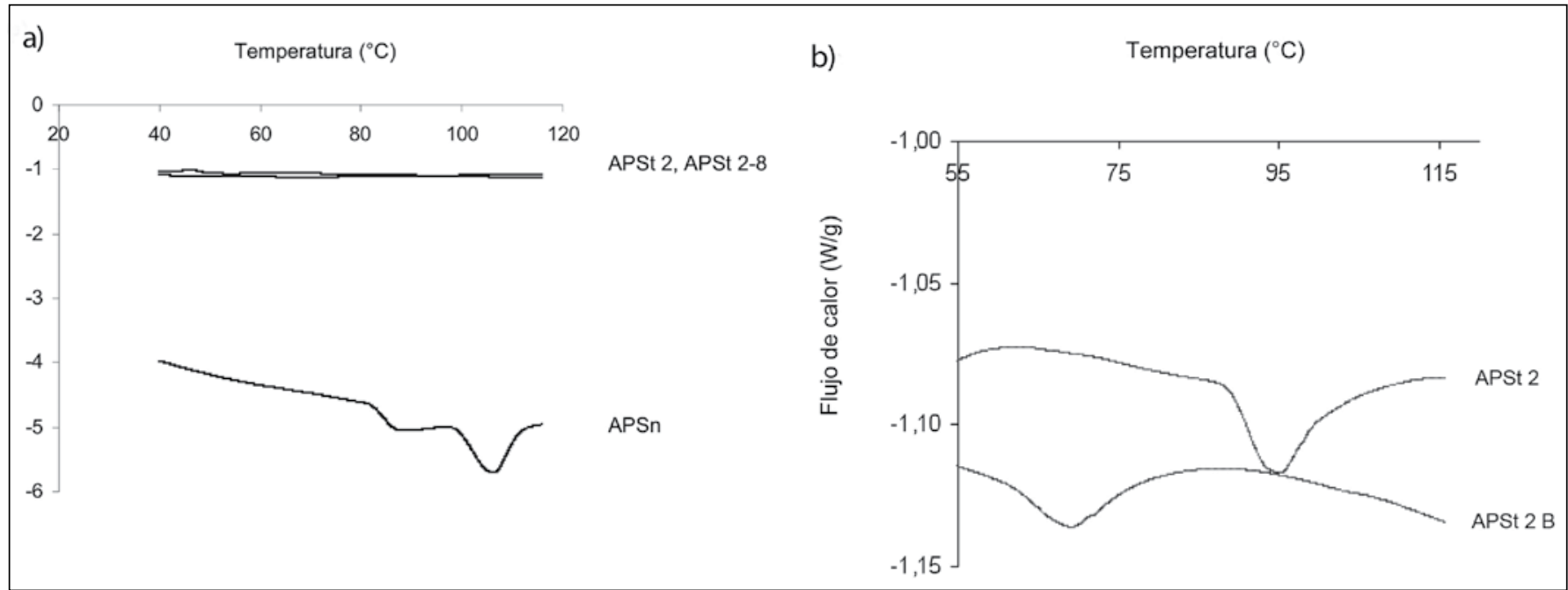

Gráfico 1. a) Termograma de APSn, APSt2 y APSt2-8. b) Termograma de APSt2 y APSt2-8.

\begin{tabular}{|c|c|c|c|c|c|c|}
\hline & VLE max (ml) & Vo $(\mathrm{ml} / \mathrm{s})$ & $\operatorname{Kgx}_{10}\left(\mathrm{ml}^{-1} / \mathrm{s}^{-1}\right)$ & $\operatorname{Kdx} 10^{4}\left(\mathrm{ml}^{-1} / \mathrm{s}^{-1}\right)$ & $\operatorname{Vg}(\%)$ & Vd (\%) \\
\hline APSn $\mathrm{pH} 8,0$ & $3,5 \pm 0,3 \mathrm{a}$ & $0,15 \pm 0,01 \mathrm{a}$ & $12,8 \pm 1,2 \mathrm{a}$ & $13 \pm 5 \mathrm{a}$ & $88 \pm 5$ & $12 \pm 5$ \\
\hline APSt2 $\mathrm{pH} 8,0$ & $7,0 \pm 0,3 \mathrm{~b}$ & $0,30 \pm 0,03 \mathrm{~b}$ & $3,3 \pm 0,7 \mathrm{~b}$ & $5 \pm 1 \mathrm{~b}$ & $92 \pm 8$ & $8 \pm 8$ \\
\hline APSt2 pH 2,5 & $8,4 \pm 0,2 \mathrm{c}$ & $0,32 \pm 0,04 \mathrm{c}$ & $3,4 \pm 0,4 \mathrm{~b}$ & $5 \pm 2 \mathrm{~b}$ & $80 \pm 3$ & $20 \pm 3$ \\
\hline
\end{tabular}

Tabla 1. kg, kd, VLEmax, Vo y contribución de los mecanismos de drenado gravitacional y desproporción de Ostwald al total de volumen de líquido drenado para las distintas muestras en distinto $\mathrm{pH}$ del medio. Los valores que se encuentran en la misma columna con la misma letra no tienen diferencias significativas $(a \leq 0,05)$.

\begin{tabular}{|l|c|c|c|} 
& $\mathbf{E}(\mathbf{m N} / \mathbf{m})$ & $\mathbf{E d}(\mathbf{m N} / \mathbf{m})$ & $\mathbf{E v}(\mathbf{m N} / \mathbf{m})$ \\
\hline APSn $\mathrm{pH} 8,0$ & $27 \pm 3 \mathrm{a}$ & $27 \pm 4 \mathrm{a}$ & $4,3 \pm 3,5 \mathrm{a}$ \\
\hline APSt2 $\mathrm{pH} 8,0$ & $24 \pm 1 \mathrm{a}$ & $23 \pm 1 \mathrm{a}$ & $7,4 \pm 0,8 \mathrm{~b}$ \\
\hline APSt2 $\mathrm{pH} 2,5$ & $41 \pm 2 \mathrm{~b}$ & $40 \pm 2 \mathrm{~b}$ & $4,3 \pm 0,4 \mathrm{a}$ \\
\hline
\end{tabular}

Tabla 2. E, Ed, Ev para las distintas muestras con diferentes condiciones de $\mathrm{pH}$. Los valores que se encuentran en la misma columna con la misma letra no tienen diferencias significativas $(a \leq 0,05)$. 


\section{Conclusiones}

El tratamiento con $\mathrm{pH}$ ácido mejora la capacidad espumante y la estabilidad de las espumas del aislado como efecto de desnaturalización de las proteínas y consecuente aumento en la hidrofobicidad superficial. La proporción de líquido drenado por escurrido gravitatorio fue significativamente superior al volumen drenado debido a la desproporción. El tratamiento ácido redujo la desestabilización por desproporción de Ostwald, lo cual sugiere la formación en la interfase de una película más resistente. APSt2 a pH 8,0 forma una película con un mayor componente viscoso, mientras que a $\mathrm{pH}$ 2,5 forma una película más elástica. Ambas condiciones favorecen la resistencia de la película a las deformaciones.

\section{Reconocimientos}

Los autores agradecen a LATU la beca otorgada por medio del convenio LATU-Facultad de Química, el soporte financiero del Programa de Desarrollo Tecnológico (PDT) del Ministerio de Educación y Cultura y el Plan de Desarrollo de las Ciencias Básicas (PEDECIBA), Uruguay.

\section{Referencias}

- CHEFTEL, J. C.; CUQ, J. L.; LORIENT, D. Proteínas alimentarias; bioquímica, propiedades funcionales, valor nutritivo, modificaciones químicas. Zaragoza: Acribia, 1989.

- DAMODARAN, S. Amino acids, peptides, and proteins. En: FENNEMA, O. R. Química de los alimentos. $3^{\mathrm{a}}$ ed. Zaragoza: Acribia, 2008.

- GEORGIEVA, D.; CAGNA, A.; LANGEVIN, D. Link between surface elasticity and foam stability. En: Soft Matter. 2009, 5(10):2063-2071.

- HAYAKAWA, S.; NAKAI, S. Relationships of hydrophobicity and net charge to the solubility of milk and soy proteins. En: Journal of Food Science. 1985, 50(2):486-491.

- KATO, A.; NAKAI, S. Hydrophobicity determined by fluorescence probe method and its correlation with surface properties of proteins. En: Biochimica et Biophysica Acta. 1980, 624(1):13-20.

- KOELSCH, P.; MOTSCHMANN, H. Relating foam lamella stability and surface dilational rheology. En: Langmuir. 2005, 21(14):6265-6269.

- LOISEL, W.; GUÉGUEN, J.; POPINEAU, Y. A new apparatus for analyzing foaming properties of proteins. En: SCHWENKE, K. D.; MOTHES, R. VCH. Food proteins, structure and functionality. Germany: Weinheim, 1993. pp. 320-323.

- LOWRY, Oliver H.; ROSEBROUGH, Nira. J.; FARR Lewis. A.; RANDALL, Rose J. Protein measurement with the folin phenol reagent. En: Journal of Biological Chemistry. 1951, 193(1):265275.

- MALDONADO-VALDERRAMA, J.; MARTÍN-MOLINA, A.; MARTÍN-RODRIGUEZ, A.; CABRERIZO-VÍLCHEZ, M. A.; GÁLVEZ-RUIZ, M. J.; LANGEVIN, D. Surface properties and foam stability of protein/surfactant mixtures: theory and experiment. En: Journal of Physical Chemistry C. 2007,111(6):2715-2723.

- MARTIN, A. H.; BOS, M. A.; VAN VLIET, T. Interfacial rheological properties and conformational aspects of soy glycinin at the air/ water interface. En: Food Hydrocolloids. 2002,16(1):63-71.

- PANIZZOLO, L. A. Modificación de proteinas por vía enzimática. Análisis de la relación estructura-funcionalidad de los productos de hidrólisis. Montevideo: UDELAR. Facultad de Química, 2005. (Tesis de Doctorado).

- PETRUCCELLI, S.; AÑÓN, M. C. The relationship between the method of preparation and the structural and functional properties of soy protein isolates. Part I: Structural and hydration properties. En: Journal of Agriculture and Food Chemistry. 1994, 42:2161-2169.

- PUPPO, M. C. Propiedades gelificantes de las proteinas de soja a pH ácido. La Plata: Universidad Nacional de La Plata. Facultad de Ciencias Exactas, 1997. (Tesis Doctoral).

- STUBENRAUCH, C.; MILLER, R. Stability of foam films and surface rheology: an oscillating bubble study at low frequencies. En: Journal of Physical Chemistry B. 2004, 108(20):6412-6421.

- UTSUMI, S.; MATSAMURA, Y.; MORI, T. Structure-function relationships of soy proteins. En: DAMORADAN, S.; PARAF, A. Food proteins and their applications. New York: Dekker, 1997.

- WAGNER, J. R.; SORGENTINI, D. A.; AÑON, M. C. Thermal and electrophoretic behavior, hydrophobity and some functional properties of acid-treatred soy isolates. En: Journal of Agricultural and Food Chemistry. 1996, 44:1881-1889.

- WAGNER, J. R. Propiedades superficiales. En: BARTHOLOMAI, W.; PILOSOF, A. M. R. eds. Caracterización funcional y estructural de proteinas. Buenos Aires: EUDEBA, 2000. 